\title{
Immune-Mediated Uveitis and Lifestyle Factors: A Review
}

\author{
Xiaoyu Guo Zhen Chen Yiqiao Xing \\ Department of Ophthalmology, Renmin Hospital of Wuhan University, Wuhan, China
}

\section{Keywords}

Immune-mediated uveitis · Smoking · Gut microbiome .

Diet $\cdot$ Physical activity

\begin{abstract}
Uveitis is a leading cause of sight-threatening disease worldwide and is characterized by inflammation of the uvea, retina, and optic nerve. Among the forms of uveitis, immunemediated uveitis represents the majority of cases in developed countries, while infectious uveitis is more common in developing countries. A Western lifestyle is thought to be one of the factors contributing to the difference and is responsible for the increasing incidence of immune-mediated uveitis. A vast range of studies have reported the importance of lifestyle factors, including smoking, gut microbiome, diet, and physical activity on immune-mediated uveitis patients and animal models of uveitis; however, there is a lack of an expert-led consensus initiative for the management of immune-mediated uveitis patients in the area of lifestyle. Herein, we summarize the advancements in the role of lifestyle factors in immune-mediated uveitis based on clinical and experimental evidence and make suggestions for patients to ameliorate inflammation and improve the prognosis, including quitting smoking, engaging in regular physical activity, consuming a personalized anti-inflammatory diet, and optimizing the gut microbiome.

(c) 2021 The Author(s).

Published by S. Karger AG, Basel
\end{abstract}

\section{Introduction}

Uveitis is a group of intraocular inflammatory diseases characterized by inflammation of the uvea and other tissues in the eye, including retina, retinal blood vessels, vitreous, and optic nerve. Uveitis is a leading cause of blindness worldwide and accounts for $10-15 \%$ of vision loss worldwide [1-3]. It is estimated that more than 2 million people worldwide suffer from uveitis and the number is increasing $[1,3]$. Uveitis can be etiologically divided into infectious uveitis and immune-mediated uveitis. The former is generally more common in developing countries $[4,5]$, while the latter is dominant in developed countries [6-8].

Immune-mediated uveitis is primarily related to autoimmunity and autoinflammation. In principle, autoimmune diseases require the identification of self-antigens, while autoinflammatory diseases are independent of autoantibodies [9]. Autoinflammatory uveitis is a relatively recent concept without a clear mechanism compared with autoimmune uveitis. Generally, autoinflammatory uveitis derives from interactions between environmental microorganisms and mutations in genes controlling innate immune pathways [9]. Mutations in genes controlling innate immune pathways make the individual more sensitive to the immune response caused by foreign organisms and the subsequent development of inflammation. Behcet's disease, tubulointerstitial ne-
This is an Open Access article licensed under the Creative Common Attribution-NonCommercial-4.0 International License (CC BY-NC) (http://www.karger.com/Services/OpenAccessLicense), applicable to the online version of the article only. Usage and distribution for commercial purposes requires written permission.
Correspondence to:

Yiqiao Xing, yiqiao_xing57@whu.edu.cn 
phritis/uveitis syndrome, and juvenile idiopathic arthritis-associated anterior uveitis are suspected to develop in this way [9].

Because great effort has been made to treat experimental autoimmune uveitis (EAU), the immunological mechanisms of autoimmune uveitis are relatively specific. Autoimmune uveitis is believed to be mediated by the differentiation of naïve CD4+ T-cells to Th1 lymphocytes and Th17 lymphocytes resulting from interactions between genetic and environmental factors [10-12]. Considering the specificity of the eye as a privileged site of immunity and the fact that retina-specific antigens are hidden away from inflammatory cells by the blood-retina barrier, it is now widely believed that 2 conditions are necessary for the development of autoimmune uveitis. The first condition is the activation of CD4+ T-cells specific for retinal antigens, such as retinal soluble antigen (S-Ag) and interphotoreceptor retinoid-binding protein (IRBP), in the periphery, in which individuals with mutations controlling the innate immune system tend to be more likely to develop autoimmune uveitis. The second is the breach of the blood-retina barrier so that the activated $\mathrm{T}$-cell act on the retina antigens. Regarding the existence of blood-retina barrier, it is more complicated to study the interaction between industrialized factors and autoimmune uveitis compared with autoimmune diseases located in other organs. The available evidence suggests that lifestyle factors can promote the progression of autoimmune uveitis through 2 conditions mentioned above.

Compared with the extent of genetic research, studies of environmental risk factors have received limited attention in immune-mediated uveitis. Industrialization has brought great benefits to humans, including better medical conditions and improved health measures, significantly decreasing the incidence of infectious and prolonging life expectancy $[13,14]$. Industrialization also caused radical shifts in lifestyle, which is found to be related to the pathogenesis of many autoimmune diseases over the past decade $[13,15,16]$. Currently, the lifestyle, sanitation, and physical environment of developed and developing countries are quite different. One explanation for the differential proportion of uveitis has been the lifestyle hypothesis. On the one hand, the incidence of infectious uveitis is reduced by excellent sanitation, and on the other hand, changes in lifestyle greatly improve the occurrence of immune-mediated uveitis. Thus, it is not surprising that the proportion of immune-mediated uveitis in developed and developing countries is vastly different. For this review, we focused on how lifestyle factors con- tribute to immune-mediated uveitis (Fig. 1), and therapeutic strategies target the factors for immune-mediated uveitis.

\section{Tobacco Smoking and Immune-Mediated Uveitis}

Industrialization has resulted in a huge increase in tobacco production, which is accompanied by major public concern. Studies have revealed the relationship between smoking and many autoimmune diseases, including rheumatoid arthritis [17], multiple sclerosis [18], systemic lupus erythematosus [19], Graves' disease [20], and Hashimoto's thyroiditis [16].

Several studies have concluded that tobacco smoking is a significant risk factor for uveitis [21-23]. According to Lin et al. [21], smoking is a risk factor for both infectious and noninfectious uveitis, while another study found smoking is only relevant with noninfectious uveitis [22]. In a cross-sectional study on noninfectious uveitis, Roesel et al. [24] found that smoking is associated with an increased risk of uveitis activity, combined with an increased need for steroid eye drops and a higher risk for cataract and macular edema. Interestingly, smoking seems to be irrelevant with Behcet's disease [25], even nicotine was used to treat the ulcers of Behcet's patients without any ocular complications [26]. Considering available evidence is based on retrospective clinical studies, more effort remains to be done to understand which component of smoking affects this condition and how it contributes to the development of uveitis.

Tobacco smoking contains many different ingredients, including nicotine, polycyclic aromatic hydrocarbons, and the 4,000 known active compounds. Although nicotine has a protective effect in Behcet's disease and other autoimmune disease, other ingredients have proinflammatory influences through several pathways in autoimmune uveitis. Cigarette smoke extract can advance

Fig. 1. Role of lifestyle factors in autoimmune uveitis. (a) Smoking: smoking can increase the release of TNF- $\alpha$, IL-1, IL-6, and IL-8 to enhance the function of inflammatory cells to contribute to the development of uveitis. (b) Gut microbiome: the gut microbiome activates $\mathrm{T}$-cell specific for retina antigen in the gut or change the homeostasis of intestinal immune to act on the course of uveitis. (c) Diet: food antigens mimicking retina-specific autoantigens may participate in the occurrence of uveitis. (d) Physical activity: physical activity reduces the level of reactive oxygen species to ameliorate the symptom of uveitis. What's more, the lower burden of the white adipose tissue will reduce the level of IL- 6 and TNF- $\alpha$ to improve the prognosis of uveitis patients.

(For figure see next page.) 


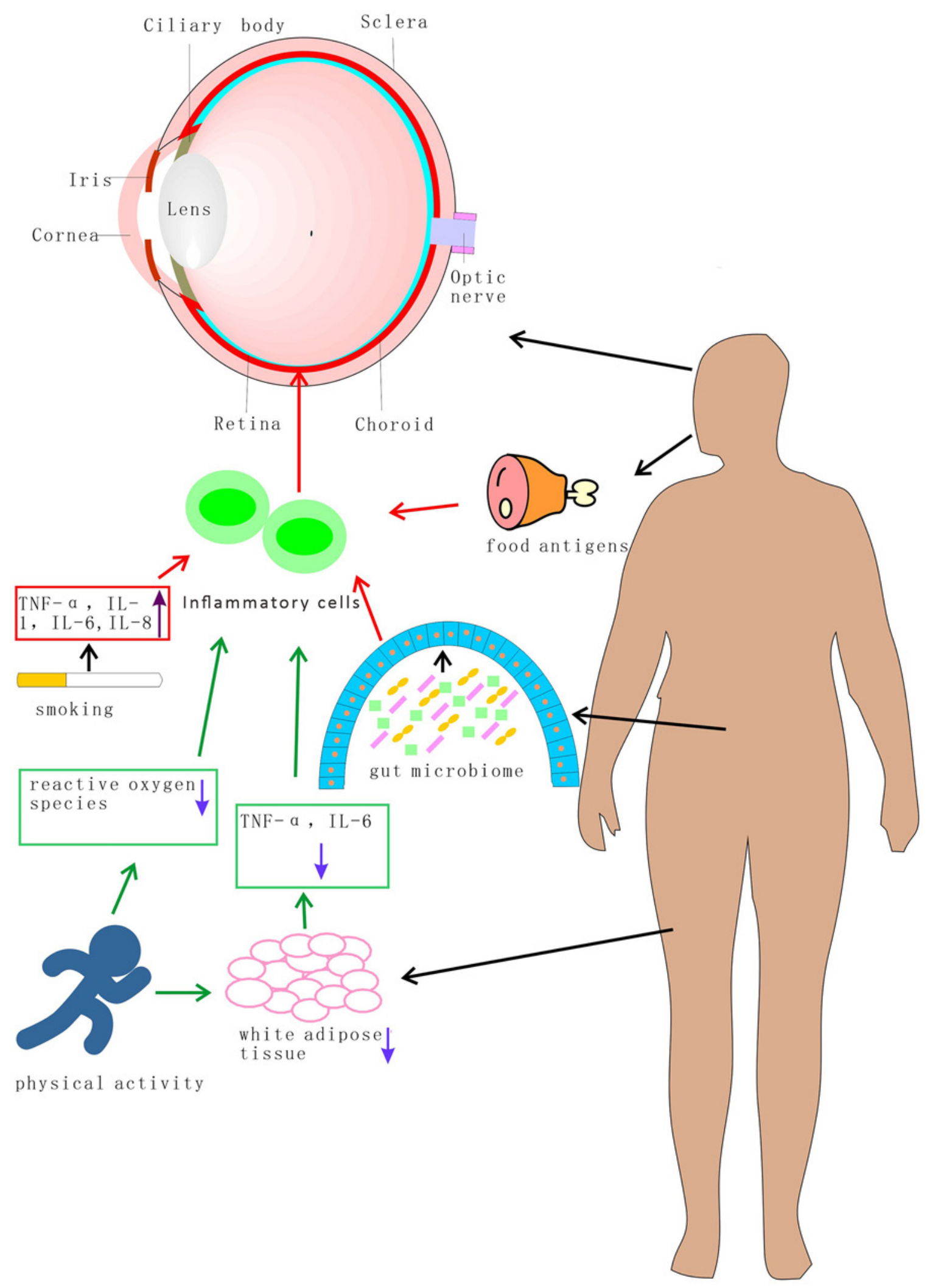


the function of inflammatory systems through NF-kB, GATA, PAX5, and Smad3/4 activation, resulting in the increased release of TNF- $\alpha$, IL-1, IL-6, and IL- 8 [27]. This activation may increase the number of inflammatory cells circulating in the blood, increasing the risks of vascular inflammation and abnormal microvascular structures, contributing to the breach of the blood-retina barrier [28]. Clinical evidence also has been found for an association between cigarette smoking and an increased risk of inflammatory macular edema, indicating that smoking causes damage to the blood-retinal barrier [24, 29, 30]. In addition to vascular inflammation, smoking or secondhand smoking exposure causes choroidal thinning in adults and children, as identified by optical coherence tomography [31-33]. Moreover, a dose-dependent effect on choroid thinning in children with exposure to secondhand smoking was reported by Yuan et al. [33]. The choroid is located between retina and sclera, and it is usually as a primary site in some types of immune-mediated uveitis, such as Vogt-Koyanagi-Harada (VKH) disease and sympathetic ophthalmitis. Changes in choroid structure may increase the risk of autoimmune uveitis located in choroid itself. Of note, the choroid mainly consists of blood vessels outside the retina and is responsible for sending oxygen and nutrients to the retina. The thinning of choroid indicates that the blood supply to the retina is abnormal and the structure around the blood-retina is more frail.

The association between smoking and immune-mediated uveitis has not been adequately examined due to a lack of experimental evidence. Exposure to cigarette smoking is a chronic process, whereas B10.RIII mice and classical animal models of EAU develop a relatively short course of disease and are immunized at an early age. Horai et al. [34] generated $R 161 H$ mice on the uveitis-susceptible B10.RIII background, a spontaneous autoimmune uveitis model without uses of complete Freund's adjuvant and IRBP. $\mathrm{R} 161 \mathrm{H}$ mice may be a more appropriate model to study it because the strain develops chronicprogressive ocular inflammation lasting $>2$ months. However, one study pointed out that exposure to smoking is similar to chronic disorders and the retina would not be affected before 5 years [31]. In addition to the duration of exposure, the complex ingredients in tobacco smoking make it more complicated. Although the mechanism by which smoking contributes to immune-mediated uveitis is obscure, quitting smoking is encouraged to reduce the recurrence and complications.

\section{Gut Microbiome and Immune-Mediated Uveitis}

The gut microbiome is composed of complex communities, intricately connected with host physiology. Our gut microbiome has accompanied us throughout the long period of human evolution, likely shaping our immune system and metabolic system [35]. Recently it has been confirmed that colonization by the gut microbiota shapes the marked B cell pool and individualized immunoglobulin repertoires, indicating the great importance of the gut microbiome to the immune system [36]. Another representative example is segmented filamentous bacteria, which can induce the differentiation of Th17 cells through the adherence to intestinal epithelial cells [37]. The gut microbiome is remodeled by changes in diet patterns and the application of antibiotics by forces of modernization. Alterations in the gut microbiome have been proven to participate in autoimmune diseases in both the intestine and extra intestine, including inflammatory bowel disease [38], multiple sclerosis [39], ankylosing spondylitis [40], rheumatoid arthritis [41], and psoriatic arthritis [42].

Information from animal models of EAU related to the gut microbiome has accumulated over the past 5 years. Two mechanisms of how the gut microbiome is involved in the development autoimmune uveitis were revealed [1]: the gut microbiome act as a trigger for activating Tcell specific for retina antigen in the gut, and [2] the gut microbiome modulate the balance of effector immune cell types (Th1 and Th17) and immunoregulatory cell types (Tregs).

Using the $\mathrm{R} 161 \mathrm{H}$ mice model, Horai et al. [43] found that an enhanced frequency of retina-specific $\mathrm{T}$ cells received an activation signal in the gut before the onset of ocular inflammation, which was independent of the endogenous source of the cognate antigen. Moreover, this activation can be stimulated by bacteria-rich intestinal contents in vitro and trigger disease in naive wild-type recipients by transfer of $\mathrm{T}$ cells cultured with extracts of intestinal contents, implying that the gut microbiome likely mimics retina antigens that are identified by the IRBP-specific TCR [43]. In addition to antigen mimics, the gut microbiome also serves as an adjuvant because oral broad-spectrum and germ-free conditions decrease the severity of disease [43]. Therefore, one or more of a particular kind of microbe plays roles in activation, while certain other kinds of microbes create pro-inflammatory surroundings for this activation. Two groups reported the modulation effect of the gut microbiome on EAU model immunized with emulsion containing complete Freund's adjuvant and IRBP peptide [44, 45]. Nakumura et al. [44] 
reported that oral broad-spectrum antibiotics administered in the short time but not intraperitoneal reduced the severity of clinical scores of B10.RIII mice through expansion of Tregs in eye-draining lymph nodes and gut-draining lymph nodes. Oral of broad-spectrum antibiotics reduced the major bacterial phyla, Firmicutes, and Bacteroidetes, as well as the bacterial class, Alphaproteobacteria [44]. Of note, the composition of the gut microbiome was significantly different between immunized and nonimmunized animals, and the diversity of gut microbiome was segregational between high clinical score animals and low score clinical animals, implying that there may exist certain types of gut microbiome that protect against or help to the course of uveitis [44]. Similar to B10.RIII mice, Heissigerova et al. [45] reported that germ-free conditions lower the gut bacterial load of $C 57 B L / 6 \mathrm{~J}$ mice to induce fewer T-cell infiltration in the retina and also fewer Th1 and Th17 numbers in the eye-draining lymph node. Both spontaneous and induced model of autoimmune uveitis all confirmed great importance of the gut microbiome in the pathogenesis of disease, whether trigger disease or modulate disease.

The exact number of gut microbes is under debate. It was generally believed over the last decade that microbes residing in the human body outnumber human cells by at least 10 -fold, while a current study find that the ratio is more close to 1:1 [46]. It still represents a large community, including bacteria, protozoa, parasitic, fungal, and viral components, residing within our body, even though the latter is more accurate. In such a large population of microbes, it remains a formidable challenge to distinguish whether activation of retina-specific $\mathrm{T}$ cells rely on 1 particular sequence of microbial component or the metabolite produced by multiple microbial interactions. Based on 16S rRNA gene and metagenomic analysis, the gut microbiome of patients with several types of uveitis was described, including acute anterior uveitis (AAU) [47], idiopathic uveitis [48], Behcet's disease [49, 50], and VKH [51]. In the clinical study of AAU, Huang et al. [47] reported a significant difference in gut microbiome beta diversity between AAU patients and controls but they did not find a significant difference in gut microbiome composition. In the study of idiopathic uveitis, several pathogenic fungi were enriched in the patients, including Malassezia restricta, Candida albicans, Candida glabrata, and Aspergillus gracilis [48]. In other studies, the unique gut microbiome characteristics of Behcet's disease and VKH patients were successfully described compared with healthy controls. Of note, a decrease in butyrate-producing bacteria was revealed in both Behcet's disease and

The Role of Lifestyle Factors in Immune-

Mediated Uveitis
VKH patients [49-51]. Butyrate is a kind of short-chain fatty acids (SCFAs) that is a metabolite of the gut microbiome and plays a protective role in maintaining host immune homeostasis. The protective effect of SCFAs on EAU was confirmed through exogenous oral SCFA administration to $C 57 \mathrm{Bl} / 6 \mathrm{~J}$ EAU mice [52]. The role of other identified microbiome remains to be elucidated in the future.

The available clinical studies have many limitations. Current studies have focused on "census taking" to distinguish the species and abundance of the microbiome. However, the host-microbe interaction is 2-way, which means that the alternation of the gut microbiome is a contributor as well as a result of autoimmune uveitis. Considering that the available evidence is speculative, it is important to clarify which microbes contribute to the development of autoimmune uveitis and which microbes protect against disease when it occurs. Moreover, the number of human gut microbiome studies in uveitis patients is small, and many subtypes of autoimmune uveitis have not been studied. To clarify the relationship between different subtypes of uveitis and the gut microbiome, more studies of higher quality, larger size, and broader scope are needed in the future. There is great significance in identifying the microbiome marker profile of gut microbiome-related uveitis and creating a beneficial gut environment by the use of fecal microbial transplantation or oral probiotics.

\section{Diet and Immune-Mediated Uveitis}

The gut is an important immunological organ that plays a unique role in the immunological responses. It is estimated that $70 \%$ of all lymphocytes are distributed in the mucosal system, a large proportion of which is the intestinal mucosal immune system [53]. As a crucial source of human nutrient intake, diet has been revealed by emerging evidence to be inextricably linked with the immune system. The close link between a Western diet and an increasing incidence of inflammatory diseases has also been widely discussed [54]. In addition to the effect on the gut microbiome mentioned above, food antigens and the pharmacological action of diet are 2 dominant mechanisms that could be responsible for the connection.

The eye, a typical immune privilege site, is traditionally thought to be separated from the immune system. However, with the further understanding of the pathogenesis of several ocular diseases, we know that the eye is not alone. The existence of gut-eye axis has been confirmed in age-related macular degeneration (AMD) [55], dry eye [56], glaucoma [57], and autoimmune uveitis 
[34]. In addition, aberrant amplitudes of ERG a-, b-wave, and oscillatory potentials were record in an animal model of experimental colitis, reflecting abnormal retinal function caused by gut inflammation [58]. This study elucidates the mechanism of uveitis as one of the common extraintestinal symptoms of patient with inflammatory bowel disease. Thus, both ocular disease and gut inflammation highlight the significance of the gut-eye axis.

Based on the gut-eye axis, the role of diet on ocular disease has been widely described in recent years. A crosssectional study reported that the advanced AMD was associated with a Western diet characterized by processed and red meats, French fries, high-fat dairy products, energy drinks, while an oriental diet composed of low glycemic index and whole-grain products contributed to a reduced risk of AMD [59]. This conclusion is also supported by experimental evidence that a high-glycemic diet, similar to a Western diet, caused symptoms of dry AMD in wild-type $C 57 \mathrm{BL} / 6 \mathrm{~J}$ mice, such as accumulation of phagosomes and lipofuscin, retinal pigment epithelium atrophy, photoreceptor cell loss, and the formation of large basal deposits [60]. Encouragingly, 2 multicenter phase III randomized clinical trials reported that a higher intake of antioxidant carotenoids, minerals, omega-3 long-chain polyunsaturated fatty acids (LCPUFAs), and $B$ vitamins and a lower intake of saturated and monounsaturated fats decreased the risk of late AMD [61]. In addition to AMD, the role of diet on glaucoma was also reported. Data from 3 prospective cohorts showed that a low-carbohydrate diet is associated with a lower risk of initial paracentral VF loss in primary open-angle glaucoma [62]. Considering the complexity of dietary components and the primary stage of the gut-eye axis, the current research is still just the tip of the iceberg.

For EAU, 2 groups reported that diet plays protective and aggravating roles in a mouse model of EAU [63-65]. Dietary intake of $\omega 3$ LCPUFAs has been shown to inhibit inflammation in a $C 57 B L / 6$ mice model of EAU [63, 64]. Interestingly, $\omega-3$ LCPUFAs not only inhibit the production of the pro-inflammatory cytokines IL-1 and IL17 mediated by dendritic cells but also reduce the amount of anti-inflammatory cytokines IL-10, but overall, the anti-inflammatory effect dominates. Another group found that a high-fat diet aggravated the severity of EAU and that melanocortin 5 receptor-deficient mice were not affected and developed EAU with a similar temporal pattern whether fed a high-fat diet or a normal-fat diet [65]. The pharmacological effects of diet are regulated by genetic factors, making the situation more complicated. The high-fat diet pattern is more similar to the Western diet pattern, and the intake of $\omega-3$ LCPUFAs usually depends on green leafy vegetables and wild fishes, which represent Mediterranean diet pattern. Experimental evidence reveals the potential hazards of the Western diet pattern and the protective effect of the Mediterranean diet pattern. Frustratingly, clinical studies on the pharmacological effects of diet on autoimmune uveitis patients are still lacking. Furthermore, TNF- $\alpha$ inhibitors have been widely used as a newly developing weapon for autoimmune disease, while a large number of patients are not sensitive to the biologics. Lifestyle is suspected to be associated with this insensitivity. As expected, a prospective cohort study is investigating whether a low intake of red and processed meat and a high-fiber diet improve the outcome of immune-mediated uveitis patients with TNF- $\alpha$ inhibitor treatment [66]. Increasing numbers of clinical studies are encouraged to fill the gap between the effects of dietary medicine and autoimmune uveitis.

Moreover, food antigens mimicking retina-specific autoantigens are a particular mechanism of autoimmune uveitis. Two environmental peptides mimicking the uveitogenic epitope from retinal S-Ag were described: "Cas" from as2casein, a common component of bovine milk, and "Rota" from surface protein vp4 from rotavirus, a common gastrointestinal pathogen [67]. Notably, oral administration of "Cas" with native cholera toxin successfully induced uveitis in rats, while S-Ag and "Rota" fail to induce the disease, although none of them could be digested. This finding supports the notion that extra-immune regulation protects the body from inflammation. In addition, the enhanced immune responses from uveitis patients to the 3 antigens compared with healthy controls indicate the pathogenic potential of food antigens. Similarly, a data-driven approach found that pigs shared unique epitopes with 40 immune-mediated diseases, including Behcet's disease [68]. The study revealed that food is a vast library of self-antigens, and taking advantage of bioinformatics approaches to find more dietary autoimmune epitopes is vital for eliminating self-antigens from diet of uveitis patients. Moreover, one special case is uveitis in celiac disease. In a case report, a glutenfree diet helped to relieve gastrointestinal symptoms and uveitis [69]. The gluten component is not relevant to retina-specific antigens, autoinflammatory response, or autoimmune response caused by antigens from other dietary sources or microbial sources stimulate T-cell migration by gastrointestinal inflammation may lead to the ocular inflammation. Effective intervention with a gluten-free diet supports those healthy gastrointestinal conditions are necessary to avoid the immune response. 
For immune-mediated uveitis tending to relapse and a prolonged duration of suffering, designing a plan including an anti-inflammatory custom diet is vital to avoid recurrence. However, current research advances cannot provide an exact answer to the question of what constitutes an anti-inflammatory diet, and further exploration will be an important direction in the future.

\section{Physical Activity and Immune-Mediated Uveitis}

It is generally believed that physical activity plays an important role in health. However, industrialization, while providing great convenience, has resulted in increased physical inactivity, which increases the prevalence of noncommunicable diseases. It is estimated that $31 \%$ of adults are physically inactive, and the ratio is even higher in developed countries [70]. Attaching increasing importance to physical activity, a recent study found an association between physical inactivity and the incidence of autoimmune diseases, such as systemic lupus erythematosus, rheumatoid arthritis, multiple sclerosis, and inflammatory bowel diseases [71].

Regular physical activity helps to reduce the level of reactive oxygen species, which are induced by pro-inflammatory cytokines to damage the uvea [72]. Finally, physical inactivity groups are more likely to be obese. In particular, the white adipose tissue acts as an essential endocrine organ secreting adipokines, including the classical cytokines IL- 6 and TNF- $\alpha$ [73]. As mentioned above, obesity can aggravate the severity of EAU though induced by high-fat diet [65]. Thus, it is not surprising that obese individuals tend to maintain a low-grade inflammatory state, and lower levers of the white adipose tissue contribute to improve the prognosis of obese patients with autoimmune uveitis. Although there is no consensus in this area, long-term regular physical activity has been shown repeatedly to improve the prognosis of patients with autoimmune diseases. In contrast, a study profiling blood components revealed that the secretion of IL- 6 and TNF- $\alpha$ increased after acute physical activity [74]. Interestingly, this surge of IL- 6 released by skeletal muscle is not the same as that in inflammation [75]. Nonetheless, it was considered prudent for patients experiencing inflammation activity to avoid physical activity. What's more, patients maintained on systemic corticosteroids need special attention because hip fracture is one of the common side effects of long-term glucocorticoid administration. Foster et al. [3] encouraged patients to perform regular, weight-bearing exercise to reduce the risk, while the initiative lacks support in the form of clinical evidence, and there is a view that weight on the hip joint should be

The Role of Lifestyle Factors in ImmuneMediated Uveitis avoided. Although there is some debate about whether patients on systemic corticosteroids should perform weight-bearing exercise, in general, physical activity is encouraged to improve the quality of life of patients in remission of inflammation and reduce the risk of recurrence of inflammation.

\section{Conclusion}

A growing body of literature highlights the influence of lifestyle factors on immune-mediated uveitis. Currently, there is a lack of expert consensus of how uveitis patients should manage their diary lives. We viewed recent advances in lifestyle factors and developed therapeutic strategies for uveitis patients to enhance their quality of life, including quitting smoking, engaging in regular physical activity, consuming a personalized anti-inflammatory diet, and optimizing the gut microbiome. Additionally, more effort will be needed to figure out the composition of an anti-inflammatory diet and the characteristic of protective gut microbiome in the future.

\section{Acknowledgments}

We thank A.J.E. for its linguistic assistance during the preparation of the manuscript.

\section{Conflict of Interest Statement}

The authors declare that there are no conflicts of interest.

\section{Funding Sources}

The authors declare no funding in this research.

\section{Author Contributions}

Y.X. made the outline of the manuscript. X.G., Z.C., and Y.X. wrote and finalized the manuscript. 


\section{References}

1 Gritz DC, Wong IG. Incidence and prevalence of uveitis in Northern California; the Northern California epidemiology of uveitis study. Ophthalmology. 2004;111(3):491-500.

2 Krishna U, Ajanaku D, Denniston AK, Gkika T. Uveitis: a sight-threatening disease which can impact all systems. Postgrad Med J. 2017; 93(1106):766-73.

3 Foster CS, Kothari S, Anesi SD, Vitale AT, Chu D, Metzinger JL, et al. The ocular immunology and uveitis foundation preferred practice patterns of uveitis management. Surv Ophthalmol. 2016;61(1):1-17.

4 Rathinam SR, Krishnadas R, Ramakrishnan R, Thulasiraj RD, Tielsch JM, Katz J, et al. Population-based prevalence of uveitis in Southern India. Br J Ophthalmol. 2011;95(4): 463-7.

5 London NJS, Rathinam SR, Cunningham ET The epidemiology of uveitis in developing countries. Int Ophthalmol Clin. 2010;50(2):1.

6 Miserocchi E, Fogliato G, Modorati G, Bandello F. Review on the worldwide epidemiology of uveitis. Eur J Ophthalmol. 2013;23(5): 705-17.

7 Thorne JE, Suhler E, Skup M, Tari S, Macaulay D, Chao J, et al. Prevalence of noninfectious uveitis in the United States: a claimsbased analysis. JAMA Ophthalmol. 2016; 134(11):1237-45.

8 Chen EJ, Bin Ismail MA, Mi H, Ho SL, Lim WK, Teoh SC, et al. Ocular autoimmune systemic inflammatory infectious study (OASIS) - report 1: epidemiology and classification. Ocul Immunol Inflamm. 2018;26(5):732-46.

9 Forrester JV, Kuffova L, Dick AD. Autoimmunity, autoinflammation, and infection in uveitis. Am J Ophthalmol. 2018;189:77-85.

10 Caspi RR. A look at autoimmunity and inflammation in the eye. J Clin Invest. 2010; 120(9):3073-83.

11 Catherine AC. Both Th1 and Th17 are immunopathogenic but differ in other key biological activities. J Immunol. 2008;180(11):7414.

12 Barry RJ, Nguyen QD, Lee RW, Murray PI, Denniston AK. Pharmacotherapy for uveitis: current management and emerging therapy. Clin Ophthalmol. 2014;8:1891-911.

13 Furman D, Campisi J, Verdin E, Carrera-Bastos $\mathrm{P}$, Targ S, Franceschi C, et al. Chronic inflammation in the etiology of disease across the life span. Nat Med. 2019;25(12):1822-32.

14 Carrera-Bastos P, O'Keefe F, Lindeberg C. The western diet and lifestyle and diseases of civilization. Res Rep Clin Cardiol. 2011.

15 Parks CG, Miller FW, Pollard KM, Selmi C, Germolec D, Joyce K, et al. Expert panel workshop consensus statement on the role of the environment in the development of autoimmune disease. Int J Mol Sci. 2014;15(8): 14269-97.
16 Miller FW, Alfredsson L, Costenbader KH, Kamen DL, Nelson LM, Norris JM, et al. Epidemiology of environmental exposures and human autoimmune diseases: findings from a national institute of environmental health sciences expert panel workshop. J Autoimmun. 2012;39(4):259-71.

17 Chang K, Yang SM, Kim SH, Han KH, Park SJ, Shin JI. Smoking and rheumatoid arthritis. Int J Mol Sci. 2014;15(12):22279-95.

18 Thompson AJ, Baranzini SE, Geurts J, Hemmer B, Ciccarelli O. Multiple sclerosis. Lancet. 2018;391(10130):1622-36.

19 Speyer CB, Costenbader KH. Cigarette smoking and the pathogenesis of systemic lupus erythematosus. Expert Rev Clin Immunol. 2018;14(6):481-7.

20 Prummel MF, Wiersinga WM. Smoking and risk of Graves' disease. JAMA. 1993;269(4): 479.

21 Lin P, Loh AR, Margolis TP, Acharya NR. Cigarette smoking as a risk factor for uveitis. Ophthalmology. 2010;117(3):585-90.

22 Yuen BG, Tham VM, Browne EN, Weinrib R, Borkar DS, Parker JV, et al. Association between smoking and uveitis: results from the pacific ocular inflammation study. Ophthalmology. 2015;122(6):1257-61.

23 Gonzalez MM, Solano MM, Porco TC, Oldenburg CE, Acharya NR, Lin SC, et al. Epidemiology of uveitis in a US population-based study. J Ophthalmic Inflamm Infect. 2018; 8(1):6.

24 Roesel M, Ruttig A, Schumacher C, Heinz C, Heiligenhaus A. Smoking complicates the course of non-infectious uveitis. Graefes Arch Clin Exp Ophthalmol. 2011;249(6):903-7.

25 Malek Mahdavi A, Khabbazi A, Yaaghoobian B, Ghojazadeh M, Agamohammadi R, Kheyrollahiyan A, et al. Cigarette smoking and risk of Behcet's disease: a propensity score matching analysis. Mod Rheumatol. 2019;29(4): 633-9.

26 Gomes JP, Watad A, Shoenfeld Y. Nicotine and autoimmunity: the lotus' flower in tobacco. Pharmacol Res. 2018;128:101-9.

27 Goncalves RB, Coletta RD, Silverio KG, Benevides L, Casati MZ, da Silva JS, et al. Impact of smoking on inflammation: overview of molecular mechanisms. Inflamm Res. 2011; 60(5):409-24

28 Grzybowski A, Nita M. Smoking and eye pathologies. a systemic review. Part I. Anterior eye segment pathologies. Current Pharmaceutical Design. 2017;23(4):629.

29 van Kooij B, Probst K, Fijnheer R, Roest M, de Loos W, Rothova A. Risk factors for cystoid macular oedema in patients with uveitis. Eye. 2008;22(2):256-60.

30 Thorne JE, Daniel E, Jabs DA, Kedhar SR, Peters GB, Dunn JP. Smoking as a risk factor for cystoid macular edema complicating intermediate uveitis. Am J Ophthalmol. 2008; 145(5):841-6.
31 Sizmaz S, Küçükerdönmez C, Pinarci EY, Karalezli A, Canan H, Yilmaz G. The effect of smoking on choroidal thickness measured by optical coherence tomography. Br J Ophthalmol. 2013;97(5):601-4.

32 Eris E, Aydin E, Ozcift SG. The effect of the smoking on choroidal thickness, central macular vascular and optic disc perfusion. Photodiagnosis Photodyn Ther. 2019;28:142-5.

33 Yuan N, Li J, Tang S, Li FF, Lee CO, Ng MPH, et al. Association of secondhand smoking exposure with choroidal thinning in children aged 6 to 8 years: the Hong Kong children eye study. JAMA Ophthalmol. 2019:1-9.

34 Horai R, Silver PB, Chen J, Agarwal RK, Chong WP, Jittayasothorn Y, et al. Breakdown of immune privilege and spontaneous autoimmunity in mice expressing a transgenic T cell receptor specific for a retinal autoantigen. J Autoimmun. 2013;44:21-33.

35 Spencer SP, Fragiadakis GK, Sonnenburg JL. Pursuing human-relevant gut microbiotaimmune interactions. Immunity. 2019;51(2): 225-39.

36 Li H, Limenitakis JP, Greiff V, Yilmaz B, Schären $\mathrm{O}$, Urbaniak C, et al. Mucosal or systemic microbiota exposures shape the B cell repertoire. Nature. 2020;584(7820):274-8.

37 Atarashi K, Tanoue T, Ando M, Kamada N, Nagano Y, Narushima S, et al. Th17 cell induction by adhesion of microbes to intestinal epithelial cells. Cell. 2015;163(2):367-80.

38 Franzosa EA, Sirota-Madi A, Avila-Pacheco J, Fornelos N, Haiser HJ, Reinker S, et al. Gut microbiome structure and metabolic activity in inflammatory bowel disease. Nat Microbiol. 2018;4(2):293-305.

39 Jangi S, Gandhi R, Cox LM, Li N, von Glehn F, Yan R, et al. Alterations of the human gut microbiome in multiple sclerosis. Nat Commun. 2016;7(1):12015

40 Wen C, Zheng Z, Shao T, Liu L, Xie Z, Le Chatelier E, et al. Quantitative metagenomics reveals unique gut microbiome biomarkers in ankylosing spondylitis. Genome Biol. 2017; 18(1): 142 .

41 du Teil Espina M, Gabarrini G, Harmsen HJM, Westra J, van Winkelhoff AJ, van Dijl JM. Talk to your gut: the oral-gut microbiome axis and its immunomodulatory role in the etiology of rheumatoid arthritis. FEMS Microbiol Rev. 2019;43(1):1-18.

42 Chimenti MS, Perricone C, Novelli L, Caso F, Costa L, Bogdanos D, et al. Interaction between microbiome and host genetics in psoriatic arthritis. Autoimmun Rev. 2018;17(3): 276-83.

43 Horai R, Zárate-Bladés CR, Dillenburg-Pilla P, Chen J, Kielczewski JL, Silver PB, et al. Microbiota-dependent activation of an autoreactive $\mathrm{T}$ cell receptor provokes autoimmunity in an immunologically privileged site. Immunity. 2015;43(2):343-53. 
44 Nakamura YK, Metea C, Karstens L, Asquith M, Gruner H, Moscibrocki C, et al. Gut microbial alterations associated with protection from autoimmune uveitis. Invest Ophthalmol Vis Sci. 2016;57(8):3747-58.

45 Heissigerova J, Seidler Stangova P, Klimova A, Svozilkova P, Hrncir T, Stepankova R, et al. The microbiota determines susceptibility to experimental autoimmune uveoretinitis. J Immunol Res. 2016;2016:5065703.

46 Sender R, Fuchs S, Milo R. Are we really vastly outnumbered? Revisiting the ratio of bacterial to host cells in humans. Cell. 2016;164(3): $337-40$.

47 Huang X, Ye Z, Cao Q, Su G, Wang Q, Deng $\mathrm{J}$, et al. Gut microbiota composition and fecal metabolic phenotype in patients with acute anterior uveitis. Invest Ophthalmol Vis Sci. 2018;59(3):1523-31.

48 Jayasudha R, Kalyana Chakravarthy S, Sai Prashanthi G, Sharma S, Tyagi M, Shivaji S. Implicating dysbiosis of the gut fungal microbiome in uveitis, an inflammatory disease of the eye. Invest Ophthalmol Vis Sci. 2019; 60(5):1384-93.

49 Consolandi C, Turroni S, Emmi G, Severgnini M, Fiori J, Peano C, et al. Behçet's syndrome patients exhibit specific microbiome signature. Autoimmun Rev. 2015;14(4):269-76.

50 Ye Z, Zhang N, Wu C, Zhang X, Wang Q, Huang $X$, et al. A metagenomic study of the gut microbiome in Behcet's disease. Microbiome. 2018;6(1):135.

51 Ye Z, Wu C, Zhang N, Du L, Cao Q, Huang $\mathrm{X}$, et al. Altered gut microbiome composition in patients with Vogt-Koyanagi-Harada disease. Gut Microbes. 2020;11:539-55.

52 Nakamura YK, Janowitz C, Metea C, Asquith M, Karstens L, Rosenbaum JT, et al. Short chain fatty acids ameliorate immune-mediated uveitis partially by altering migration of lymphocytes from the intestine. Sci Rep. 2017; 7(1):11745

53 Mueller C, Macpherson AJ. Layers of mutualism with commensal bacteria protect us from intestinal inflammation. Gut. 2006;55(2): 276-84.

54 Thorburn AN, Macia L, Mackay CR. Diet, metabolites, and "western-lifestyle" inflammatory diseases. Immunity. 2014;40(6):83342.
55 Rinninella E, Mele MC, Merendino N, Cintoni M, Anselmi G, Caporossi A, et al. The role of diet, micronutrients and the gut microbiota in age-related macular degeneration: new perspectives from the gut(-) retina axis. Nutrients. 2018;10(11):1677.

56 Trujillo-Vargas CM, Schaefer L, Alam J, Pflugfelder SC, Britton RA, de Paiva CS. The gut-eye-lacrimal gland-microbiome axis in Sjögren Syndrome. Ocul Surf. 2020;18(2): 335-44.

57 Chaiwiang N, Poyomtip T. Microbial dysbiosis and microbiota-gut-retina axis: the lesson from brain neurodegenerative diseases to primary open-angle glaucoma pathogenesis of autoimmunity. Acta Microbiol Immunol Hung. 2019;66(4):541-58.

58 Peng K, Xiao J, Wang J, Song Y, Wu L, Xiu W, et al. MAdCAM-1 mediates retinal neuron degeneration in experimental colitis through recruiting gut-homing CD4(+) T cells. Mucosal Immunol. 2021 Jan;14(1):152-63.

59 Chiu CJ, Chang ML, Zhang FF, Li T, Gensler $\mathrm{G}$, Schleicher M, et al. The relationship of major American dietary patterns to age-related macular degeneration. Am J Ophthalmol. 2014;158(1):118-e1.

60 Rowan S, Jiang S, Korem T, Szymanski J, Chang ML, Szelog J, et al. Involvement of a gut-retina axis in protection against dietary glycemia-induced age-related macular degeneration. Proc Natl Acad Sci USA. 2017; 114(22):E4472-E81.

61 Agron E, Mares J, Clemons TE, Swaroop A, Chew EY, Keenan TDL, et al. Dietary nutrient intake and progression to late age-related macular degeneration in the age-related eye disease studies 1 and 2. Ophthalmology. 2021 Mar;128(3):425-42.

62 Hanyuda A, Rosner BA, Wiggs JL, Willett WC, Tsubota K, Pasquale LR, et al. Low-carbohydrate-diet scores and the risk of primary open-angle glaucoma: data from three US cohorts. Eye. 2020;34(8):1465-75.

63 Shoda H, Yanai R, Yoshimura T, Nagai T, Kimura K, Sobrin L, et al. Dietary omega-3 fatty acids suppress experimental autoimmune uveitis in association with inhibition of Th1 and Th17 cell function. PLoS One. 2015; 10(9):e0138241.

64 Uchi SH, Yanai R, Kobayashi M, Hatano M, Kobayashi Y, Yamashiro C, et al. Dendritic cells mediate the anti-inflammatory action of omega-3 long-chain polyunsaturated fatty acids in experimental autoimmune uveitis. PLoS One. 2019;14(7):e0219405.
65 Muhammad FY, Peters K, Wang D, Lee DJ. Exacerbation of autoimmune uveitis by obesity occurs through the melanocortin 5 receptor. J Leukoc Biol. 2019;106(4):879-87.

66 Christensen R, Heitmann BL, Andersen KW, Nielsen OH, Sørensen SB, Jawhara M, et al Impact of red and processed meat and fibre intake on treatment outcomes among patients with chronic inflammatory diseases: protocol for a prospective cohort study of prognostic factors and personalised medicine. BMJ Open. 2018;8(2):e018166.

67 Wildner G, Diedrichs-Möhring M. Autoimmune uveitis and antigenic mimicry of environmental antigens. Autoimmun Rev. 2004; 3(5):383-7.

68 Gershteyn IM, Ferreira LMR. Immunodietica: a data-driven approach to investigate interactions between diet and autoimmune disorders. J Transl Autoimmun. 2019;1:100003.

69 Klack K, Pereira RM, de Carvalho JF. Uveitis in celiac disease with an excellent response to gluten-free diet: third case described. Rheumatol Int. 2011;31(3):399-402.

70 Hallal PC, Andersen LB, Bull FC, Guthold R, Haskell W, Ekelund U. Global physical activity levels: surveillance progress, pitfalls, and prospects. Lancet. 2012;380(9838):247-57.

71 Sharif K, Watad A, Bragazzi NL, Lichtbroun M, Amital H, Shoenfeld Y. Physical activity and autoimmune diseases: get moving and manage the disease. Autoimmun Rev. 2018; 17(1):53-72.

72 Kruk J, Kubasik-Kladna K, Aboul-Enein YH The role oxidative stress in the pathogenesis of eye diseases: current status and a dual role of physical activity. Mini Rev Med Chem. 2015; 16(3):241

73 Versini M, Jeandel PY, Rosenthal E, Shoenfeld Y. Obesity in autoimmune diseases: not a passive bystander. Autoimmun Rev. 2014; 13(9):981-1000.

74 Contrepois K, Wu S, Moneghetti KJ, Hornburg D, Ahadi S, Tsai MS, et al. Molecular choreography of acute exercise. Cell. 2020; 181(5):1112-e16.

75 Nieman DC, Nehlsen-Cannarella SL, Fagoaga OR, Henson DA, Utter A, Davis JM, et al. Influence of mode and carbohydrate on the cytokine response to heavy exertion. Med Sci Sports Exerc. 1998;30(5):671-8. 